\title{
Clinical utility of eslicarbazepine: current evidence
}

This article was published in the following Dove Press journal:

Drug Design, Development and Therapy

10 February 2015

Number of times this article has been viewed

\author{
Gaetano Zaccara' \\ Fabio Giovannelli', ${ }^{1,2}$ \\ Massimo Cincotta' \\ Alessia Carelli ${ }^{3}$ \\ Alberto Verrotti ${ }^{3}$ \\ 'Department of Medicine, Unit \\ of Neurology, Florence Health \\ Authority, Florence, Italy; \\ ${ }^{2}$ Department of Neuroscience, \\ Psychology, Pharmacology and \\ Child Health (NEUROFARBA), \\ University of Florence, Florence, Italy; \\ ${ }^{3}$ Department of Pediatrics, University \\ of Perugia, Perugia, Italy
}

Correspondence: Gaetano Zaccara Unit of Neurology, San Giovanni di Dio Hospital, Via Di Torregalli n 3, 50143 Florence, Italy Email gaetano.zaccara@asf.toscana.it
Abstract: Eslicarbazepine acetate (ESL) is a new antiepileptic drug whose mechanism of action is blockade of the voltage-gated sodium channel (VGSC). However, in respect to carbamazepine and oxcarbazepine, the active ESL metabolite (eslicarbazepine) affects slow inactivation of VGSC and has a similar affinity for the inactivated state and a lower affinity for the resting state of the channel. This new antiepileptic drug has been recently approved in Europe (trade name Zebinix) and in the United States (trade name Stedesa) for adjunctive treatment in adult subjects with partial-onset seizures, with or without secondary generalization. Following oral administration, ESL is rapidly and extensively metabolized by hepatic esterases to eslicarbazepine. This active metabolite has a linear pharmacokinetic profile, a low binding to plasma proteins ( $<40 \%$ ), and a half-life of $20-24$ hours and is mainly excreted by kidneys in an unchanged form or as glucuronide conjugates. ESL is administered once a day and has a low potential for drug-drug interactions. Efficacy and safety of this drug in patients with focal seizures have been assessed in four randomized clinical trials, and responder rates (percentage of patients with a $\geq 50 \%$ improvement of their seizures) ranged between $17 \%$ and $43 \%$. Adverse events were usually mild to moderate, and the most common were dizziness, somnolence, diplopia, abnormal coordination, blurred vision, vertigo, headache, fatigue, nausea, and vomiting. ESL may be considered an interesting alternative to current antiepileptic drugs for the treatment of drug-resistant focal epilepsies. Additionally, it is under investigation in children with focal epilepsies, in patients with newly diagnosed focal epilepsies, and also in other neurological and psychiatric disorders.

Keywords: antiepileptic drugs, epilepsy, eslicarbazepine acetate, pharmacoresistant epilepsy, oxcarbazepine, carbamazepine

\section{Introduction}

Epilepsy has an annual incidence of about 50 per 100,000 and a prevalence between 5 and 10 per $1,000 .{ }^{1}$ Treatment of this common neurological disorder is mainly symptomatic, with antiepileptic drugs (AEDs) considered to be the main therapeutic approach. However, despite a broad range of commonly used AEDs, approximately $30 \%$ of adult patients with epilepsy have their seizures uncontrolled. ${ }^{2}$ Most importantly, adverse effects are a major deterrent to successful treatment with AEDs. In patients with epilepsy, it has been shown that they negatively impact on health-related quality of life, are a significant source of disability, and morbidity, ${ }^{3-5}$ and may lead to treatment discontinuation in up to $25 \%$ of patients $^{6-8}$ or a low adherence to the treatment. ${ }^{8,9}$

Eslicarbazepine acetate (ESL) is a third-generation member of the dibenzazepine family, which also includes carbamazepine (CBZ) and oxcarbazepine (OXC). ${ }^{10-13}$ It has been approved in 2009 by the European Medicines Agency and in 2013 by the US Food and Drug Administration as adjunctive therapy in adults with partial-onset seizures with or without secondary generalization. It is available in Europe in the pharmaceutical form of tablets under the trade name Zebinix (produced by BIAL, Trofa, Porto District, Portugal) and in the United States under the trade name Stedesa 
(produced by Sunovion Pharmaceuticals, Marlborough, MA, USA).

In respect to $\mathrm{CBZ}$ and $\mathrm{OXC}, \mathrm{ESL}$ may be used with a simplified drug regimen, and there are some data that suggest an improved tolerability profile.

\section{Mechanisms of action and pharmacokinetics Mechanisms of action}

All AEDs exert their effect through a reduction of membrane excitability, which is the consequence of interaction with ion channels or neurotransmitter receptors. Blockade of voltagegated sodium channel (VGSC) is the proposed mechanism of action for various AEDs, such as CBZ, lamotrigine, OXC, and phenytoin, ${ }^{14}$ and ESL shares with these AEDs the characteristic of being a potent blocker of VGSC. ${ }^{10}$

It has been recently shown that there are some differences between AEDs in the way this effect is achieved. VGSC has three distinctive states: resting state, open state, and inactivated state. During the resting or deactivated phase, the VGSC is closed, but it opens in response to a depolarization impulse. This allows the entrance of sodium ion into the cell and generates the action potential. Immediately after the action potential, the channel goes for a few milliseconds into inactivated state, in which it is closed again but cannot be opened because it is not responsive to voltage changes. ${ }^{15}$

The suggested mechanism for those AEDs acting on VGSC is to prolong the inactivated state of the sodium channel, thus preventing its reversion to the resting form and a possible further depolarization. The consequence is that they limit sustained repetitive neuronal firing. ${ }^{11,16,17}$

However, the affinity of a drug for the channel may be different in different functional states of the channel, and different VGSC blockers may behave differently. In fact, the affinity of ESL for the inactivated state of the VGSC is similar to that of CBZ, while the affinity of ESL for the resting state of the channel is about three times lower than that of CBZ. As a consequence, ESL should be equally effective in preventing sustained repetitive neural firing but less effective in disturbing physiological mechanisms. ${ }^{18}$

Other important differences have been recently demonstrated between AEDs acting on VGSC. It is known that there are at least two distinct kinetic classes of inactivation, termed fast and slow.

Within milliseconds of depolarization, the channels enter the fast-inactivated state which is characterized by closure of the channel pore, which does not reopen until the cell is hyperpolarized. ${ }^{19}$ During fast inactivation, a cytoplasmic region (the inactivating particle) occludes the pore ${ }^{20}$ and makes the cell refractory to firing. ${ }^{20,21}$ Slow inactivation is a separate process that appears after prolonged depolarization, does not involve the inactivating particle, and may result from a structural rearrangement of the pore. ${ }^{19}$ Fast inactivation of $\mathrm{Na}$ channels is thought to contribute to action potential termination and regulation of the refractory period, ${ }^{22}$ while slow inactivation, which occurs on a much slower time scale of seconds, is thought to contribute to overall membrane excitability by increasing action potential thresholds, thereby limiting action potential burst durations and, most importantly, their propagation within dendrites..$^{23,24}$

Although the majority of VGSC blockers used in the treatment of epileptic seizures interfere with the fast inactivation pathway, there is information that suggests that some AEDs may influence the slow inactivation of VGSC. ${ }^{20}$ For example, lacosamide (LCM) was shown to act by enhancing slow inactivation of VGSC. ${ }^{25}$

Recently, the effect of ESL, CBZ, OXC, and LCM on the fast and slow inactivated states of VGSCs has been evaluated in an experimental study, ${ }^{26}$ and it has been observed that both ESL and LCM reduce VGSC availability through enhancement of slow inactivation, while these AEDs do not share with $\mathrm{CBZ}$ and $\mathrm{OXC}$ the ability to alter fast inactivation of VGSC.

In conclusion, ESL is a VGSC blocker which, in respect to $\mathrm{CBZ}$, has a similar affinity for inactivated state of the channel and a lower affinity for the resting state. Furthermore, in respect to $\mathrm{CBZ}$ and $\mathrm{OXC}$, ESL affects slow inactivation and not fast inactivation of VGSC. Both these characteristics would suggest similar effect on sustained repetitive neural firing with a lower propensity in disturbing physiological mechanisms.

In animal models, ESL is effective against seizures induced by proconvulsant agents such as metrazole, bicuculline, 4-aminopyridine, and picrotoxin, ${ }^{11,27,28}$ and is also effective in the amygdala-kindled rat model. ${ }^{11}$ In these models, ESL was equipotential to $\mathrm{CBZ}$ and more potent than $\mathrm{OXC}$, showed less neurological impairment in rats, and was less toxic to cultured hippocampal neurons. ${ }^{29}$ The ESL activity profile in animal models predicts efficacy in partial and generalized tonic-clonic seizures in humans. ${ }^{11}$

\section{Pharmacokinetics}

Following oral administration, absorption of ESL from the gastrointestinal tract is high. In fact, it has been shown that 
the amount of metabolites recovered in urine corresponded to more than $90 \%$ of the administered ESL dose. ${ }^{12}$ After the absorption, the drug is rapidly and extensively metabolized by hepatic esterases to eslicarbazepine (also known as $S$-licarbazepine), which is the major ESL metabolite and is responsible for the pharmacological effect. ${ }^{12}$ As a consequence, plasma ESL concentration always remains below the limit of detection (Table 1). ${ }^{11}$

Concomitant intake of ESL with food does not influence absorption. ${ }^{30}$ Peak plasma eslicarbazepine concentrations are attained at 2-3 hours post-dose, and steady-state plasma concentrations are attained after 4-5 days of once-daily dosing, consistent with an effective half-life in the order of 20-24 hours. ${ }^{12,31,32}$

Binding of eslicarbazepine to plasma proteins is relatively low $(<40 \%)$ and is not affected by other coadministered drugs. ${ }^{11,32}$

A minor ESL metabolite in plasma is the right isomer of eslicarbazepine, $R$-licarbazepine, which is inactive. A small amount of both stereosomers, eslicarbazepine and $R$-licarbazepine, undergo chiral inversion through metabolic oxidation to $\mathrm{OXC} .^{33}$

ESL does not affect its own metabolism, ${ }^{34}$ and its pharmacokinetic parameters are dose-proportional across the dose range of 400-2,400 mg/d in healthy subjects ${ }^{35}$ and in patients. ${ }^{36}$ Finally, ESL and its metabolites are eliminated from the systemic circulation primarily by renal excretion, and approximately two-thirds of them are in the unchanged form and one-third as glucuronide conjugates.

\section{Special populations}

ESL pharmacokinetic profile has been specifically assessed in children and adolescents, ${ }^{36}$ and no relevant differences have been found in respect to adults, although dose-normalized $C_{\max }$ and AUC depended on age, indicating faster

Table I Pharmacokinetic and metabolism of the active metabolite eslicarbazepine

\begin{tabular}{ll}
\hline$T_{\text {max }}$ & $2-3 \mathrm{~h}$ \\
Bioavailability & High \\
$T_{1 / 2}$ & $20-24 \mathrm{~h}$ \\
Pharmacokinetics & Linear over the dose range of $400-2,400 \mathrm{mg} / \mathrm{d}$ \\
Protein binding & $<40 \%$ \\
Metabolism & Minor metabolites in plasma are $R$-licarbazepine \\
& and oxcarbazepine. Other minor metabolites \\
& are glucuronic acid conjugates of eslicarbazepine, \\
& R-licarbazepine, and oxcarbazepine. \\
Excretion & Urinary excretion \\
\hline
\end{tabular}

Notes: Data from Almeida and Soares-da-Silva" and Bialer and Soares-da-Silva. ${ }^{12}$ Abbreviations: $T_{\max }$, time to achieve peak concentration; $T_{1 / 2}$, half life. clearance in younger children than in adolescents. ${ }^{12}$ The pharmacokinetic profile of ESL is not significantly affected by old age ${ }^{32}$ and sex. ${ }^{37}$

The effect of renal function on ESL pharmacokinetics has been studied in patients with mild, moderate, and severe renal function impairments, ${ }^{38}$ and a significant relationship has been found between creatinine clearance $(\mathrm{CrCl})$ and eslicarbazepine renal clearance. The excretion of other ESL metabolites (eslicarbazepine-glucuronide, $(R)$-licarbazepine, $(R)$-licarbazepine-glucuronide, $\mathrm{OXC}$, and OXC-glucuronide) is also significantly affected by kidney diseases. Values of creatinine clearance between 30 and $60 \mathrm{~mL} / \mathrm{min}$ should require half the dose, while data are insufficient to establish a recommendation for dose adjustment in patients with $\mathrm{CrCl}<30 \mathrm{~mL} / \mathrm{min} .{ }^{38}$ Finally, hemodialysis partially removes ESL from plasma. ${ }^{32}$

Liver function has less critical effects on ESL kinetics. In patients with a moderate liver impairment, ESL pharmacokinetics was not significantly affected, although there were more subjects with measurable plasma ESL concentrations in the hepatic impairment group than in the control group. ${ }^{39}$ Biotransformation of ESL to eslicarbazepine during first passage does not seem to be affected by portal-systemic shunting. Glucuronide metabolites are also unchanged in patients with moderate liver disease. ${ }^{39}$

\section{Drug interactions}

In vitro, ESL is a weak inducer of cytochrome P450 3A4 (CYP3A4) and UDP-glucuronyl transferases. Thus, an increase in the dose of the medical products that are mainly metabolized through CYP3A4 or through the UDP-glucuronyl transferases may be required when coadministered with ESL. ${ }^{12,32}$ ESL has also inhibiting properties with respect to CYP2C19, which might require dose adjustments of coadministered drugs metabolized by this enzyme (eg, phenytoin, clopidogrel, omeprazole, and diazepam). ${ }^{12}$

\section{Interactions with other AEDs}

Data from healthy subjects have shown that concomitant administration of ESL 1,200 mg once daily with the enzyme inducers phenytoin or CBZ resulted in an average decrease of $31 \%-33 \%$ in exposure to eslicarbazepine, most likely caused by an induction of glucuronidation. ${ }^{12} \mathrm{On}$ the basis of individual response, the dose of ESL may need to be increased when coadministered with these drugs. An average increase of 31\%-35\% in exposure to phenytoin, most likely caused by an inhibition of CYP2C19, has also been observed in these patients, which may require a reduction of phenytoin dose..$^{12,32}$ 
Similar studies on healthy volunteers showed minor pharmacokinetic interactions between ESL and lamotrigine, and ESL and topiramate (with a 15\% reduction to the exposure to lamotrigine and an $18 \%$ reduction to the exposure to topiramate). ${ }^{12}$

A population pharmacokinetic analysis in epileptic adult patients has indicated that a concomitant administration of this agent with valproate or levetiracetam does not affect the exposure to eslicarbazepine..$^{32,40}$

\section{Interactions with other medicinal products}

Administration of ESL 1,200 mg once daily to female subjects using a combined oral contraceptive showed an average decrease of $37 \%$ and $42 \%$ in systemic exposure to levonorgestrel and ethinylestradiol, respectively, most likely caused by an induction of CYP3A4. Therefore, women of childbearing potential should use other adequate methods of contraception during treatment with ESL. ${ }^{41}$

A reduction of systemic exposure to simvastatin and rosuvastatin (50\% and 36\%-39\%, respectively) has been observed in healthy subjects when ESL is coadministered with these statins. ${ }^{12,32}$

Coadministration of ESL with warfarin also was found to cause a mild (23\%) but statistically significant decrease in $(S)$-warfarin plasma exposure, with no significant effect on the $(R)$-warfarin pharmacokinetics or coagulation. ${ }^{42}$ However, due to interindividual variability in the interaction, special attention should be directed to INR (international normalized ratio) monitoring during the first weeks of initiation or at the end of concomitant warfarin-ESL treatment. ${ }^{33}$ No interaction has been observed between digoxin and ESL in healthy volunteers. ${ }^{12}$

Since ESL and OXC exert their effects through an identical metabolite, which is eslicarbazepine, it may be important to underline kinetic differences between these two AEDs.

The first difference is that, after OXC administration, a peak concentration of the parent drug is detectable in plasma, with a half-life of $1-2.5$ hours, ${ }^{43}$ and also in the cerebrospinal fluid. ${ }^{44}$ This OXC peak concentration may produce an effect in brain before transformation to its active metabolite (it should be remembered that OXC affects fast inactivation of VGSC). In contrast, after ESL administration, the agent is rapidly converted to eslicarbazepine and is not even detectable in plasma. ${ }^{18,31}$

A further point is that $\mathrm{OXC}$ is transformed to equal amounts of both stereoisomers of which one is active (eslicarbazepine) and the other is inactive ( $R$-licarbazepine), whereas ESL is mainly transformed to the active metabolite.
This results in a $40 \%$ increase in the delivery of the active metabolite eslicarbazepine into the plasma as well as a significantly lower systemic exposure to the inactive $(R)$-licarbazepine when compared with OXC. ${ }^{35}$

\section{Experiences in adults: efficacy, tolerability, and safety}

For the assessment of ESL efficacy and tolerability as an add-on drug in focal epilepsies, 1,192 adult patients with drug-resistant focal epilepsies were recruited in one Phase $\mathrm{II}^{45}$ and three Phase III $^{46-48}$ studies. In all studies, patients with at least four simple or complex partial-onset seizures per month despite treatment with 1-2 or 1-3 AEDs could be recruited.

In the Phase II study, ${ }^{45} 143$ patients were randomized to one of three groups: treatment with ESL once daily $(n=50)$, twice daily $(n=46)$, or placebo $(n=47)$. In patients treated with the active drug, ESL dose was titrated up to 1,200 mg/d in three steps at 4-week intervals. A statistically significant difference (from the placebo group) in the percentage of responders was noted only for the once-daily group and not for the twice-daily group, and the incidence of adverse events was similar between the treatment groups, with no drug-related serious adverse events occurring. On the basis of these results, ESL has been administered once daily in all other studies.

Main characteristics and results of the three Phase III double-blind, add-on studies are reported in Table 2. In these studies, ESL was started with a dose of 400 or $800 \mathrm{mg}$ once daily, and dose increments to $1,200 \mathrm{mg} / \mathrm{d}$ were performed after 1 week or more slowly.

Total duration of the double-blind phase was 12 or 14 weeks. ${ }^{46-48}$ Efficacy end points were proportion of responders (proportion of patients with $\geq 50 \%$ decrease in seizure frequency) and the median relative reduction in standardized seizure frequency. Results of these studies have also been pooled. ${ }^{49}$

Overall, results of these studies clearly show that oncedaily ESL, at a dose of 800 or 1,200 mg/d, is efficacious and well tolerated as adjunctive therapy in drug-resistant focal epilepsies. Incidentally, in the pooled analysis it was noted that the incidence of AEs was higher in those patients in whom ESL had been added to CBZ. ${ }^{49}$

Two open-label extension studies have been performed ${ }^{50,51}$ as part of the ESL clinical development program in patients who completed registrative Phase III studies. ${ }^{46,48}$ The primary objective of these studies was to evaluate the long-term tolerability and safety of once-daily adjunctive ESL, and the 
Table 2 Main characteristics and results of Phase III, double-blind studies performed with eslicarbazepine acetate, in patients with drug-resistant focal epilepsies

\begin{tabular}{|c|c|c|c|c|c|c|}
\hline Reference & Age & $\begin{array}{l}\text { AED } \\
\text { allowed }\end{array}$ & Titration speed & $\begin{array}{l}\text { Randomized } \\
\text { patients }\end{array}$ & $\begin{array}{l}\text { Responder } \\
\text { rate }^{\mathrm{a}}\end{array}$ & $\begin{array}{l}\text { Median percent change } \\
\text { in seizure frequency (\%) }\end{array}$ \\
\hline \multirow[t]{5}{*}{ Elger et $\mathrm{al}^{46}$} & $18-76$ & $1-2$ & Starting dose: $400 \mathrm{mg} / \mathrm{d}$ & $n=402$ & & \\
\hline & & & Dose increments of & Placebo $=102$ & 20.0 & 16.0 \\
\hline & & & $400 \mathrm{mg} / \mathrm{d}$ each week & $\mathrm{ESL}, 400 \mathrm{mg}=100$ & $23.0 *$ & 26.0 \\
\hline & & & & $\mathrm{ESL}, 800 \mathrm{mg}=98$ & $34.0 * *$ & $36.0^{*}$ \\
\hline & & & & $\mathrm{ESL}, \mathrm{I}, 200 \mathrm{mg}=102$ & $43.0 * *$ & $45.0 * *$ \\
\hline \multirow[t]{4}{*}{ Gil Nagel et al ${ }^{47}$} & $17-77$ & $1-2$ & Starting dose: $400 \mathrm{mg} / \mathrm{d}$ & $n=252$ & & \\
\hline & & & Final dose achieved within $2 \mathrm{wk}$ & Placebo $=87$ & 22.6 & 17.0 \\
\hline & & & & $\mathrm{ESL}, 800 \mathrm{mg}=85$ & 34.5 & $37.9 * * *$ \\
\hline & & & & $\mathrm{ESL}, \mathrm{I}, 200 \mathrm{mg}=80$ & $37.7^{* * *}$ & $41.9 * * *$ \\
\hline \multirow[t]{5}{*}{ Ben-Menachem et $\mathrm{al}^{48}$} & $18-69$ & $1-3$ & Starting dose: $400 \mathrm{mg}$ or $800 \mathrm{mg} / \mathrm{d}$ & $n=395$ & & \\
\hline & & & Pts randomized to $1,200 \mathrm{mg} / \mathrm{d}$ & Placebo $=100$ & 13.0 & 0.8 \\
\hline & & & achieved final dose within $2 \mathrm{wk}$ & $\mathrm{ESL}, 400 \mathrm{mg}=96$ & 17.0 & 18.7 \\
\hline & & & & $E S L, 800 \mathrm{mg}=|0|$ & $40.0 * *$ & $32.6 * *$ \\
\hline & & & & $\mathrm{ESL}, \mathrm{I}, 200 \mathrm{mg}=98$ & $37.1^{* *}$ & $32.8^{* *}$ \\
\hline
\end{tabular}

Notes: aProportion of patients with $\geq 50 \%$ decrease in seizure frequency. $* P<0.05 ; * * P<0.00$ I active drug vs placebo (titration + maintenance vs baseline); $* * * P<0.05$ active drug vs placebo (maintenance vs baseline).

Abbreviations: AED, antiepileptic drug; ESL, eslicarbazepine acetate; Pts, patients.

secondary objective was to assess whether the efficacy of this drug was maintained with long-term use.

In these studies the starting dose was once-daily $800 \mathrm{mg}$ for 4 weeks, and, after that, the dosage could be titrated down or up between 400 and 1,200 $\mathrm{mg}$ to individualize therapy, while doses of concomitant AEDs had to be kept stable. Of a total of 314 and 325 patients who were enrolled after completing previous double-blind trials, ${ }^{46,48} 239$ and 223 completed 1 year of open treatment.

Sustained therapeutic effect and favorable tolerability and safety profile were reported in both the long-term studies. Interestingly, in this selected population of patients, the proportion of patients who achieved seizure freedom for a 12 -week period ranged between $8.7 \%$ and $12.5 \%$ for the first study ${ }^{50}$ and $5 \%$ and $11 \%$ for the second, ${ }^{51}$ and improvements in the quality of life and depressive symptoms were observed.

Several recent retrospective studies have confirmed the efficacy and safety of ESL in populations of patients with focal epilepsies of different severity. ${ }^{52-55}$

Owing to the heterogeneity of casistics and the retrospective nature of these studies, assessments of efficacy could not be done. However, it is confirmed that the tolerability spectrum of ESL is generally favorable. Retention analyses of these heterogeneous studies showed withdrawal rates of $24.6 \%$ at 3 months, ${ }^{52}$ of $11.5 \%$ at 6 months, ${ }^{53} 27.6 \%$ at 1 year, ${ }^{54}$ and $34.9 \%$ at 2 years. ${ }^{55}$ In some of these studies, ESL was added to or replaced with $\mathrm{CBZ}$ or OXC, and it was noted that switching from OXC to ESL at a dose ratio of 1:1 was apparently associated with a better tolerability during ESL treatment, while switching from CBZ to ESL did not reduce adverse effects..$^{55}$ It was also noted that ESL efficacy was better in subjects in whom ESL was not coadministered with other sodium channel blockers ${ }^{54}$ and mainly with LCM. ${ }^{53}$ All these findings should be taken with extreme caution and should possibly be confirmed by further studies.

The tolerability pattern of ESL may be in part analyzed in registrative, double-blind studies which allow the assessment of frequent, dose-dependent adverse effects. The most often reported adverse events in clinical trials were neurological, with somnolence, dizziness, vertigo, ataxia, abnormal coordination, diplopia, fatigue, headache being the most frequent. ${ }^{49}$

Recently, a network meta-analysis has compared the tolerability of ESL, LCM, and OXC in drug-resistant epilepsies from double-blind, placebo-controlled trials. At high recommended doses, patients treated with $\mathrm{OXC}$ withdrew from the experimental treatment significantly more frequently than did patients treated with ESL and LCM. Furthermore, some vestibulo-cerebellar adverse effects (coordination abnormal/ ataxia and diplopia) were significantly more frequently observed in patients treated with OXC than in patients treated with LCM and ESL. ${ }^{56}$

Behavioral and psychiatric disturbances (eg, agitation, anxiety, depression, and even suicide ideation) are a frequent complaint in patients with drug-resistant focal epilepsies, which in some cases can be related to pharmacological treatment. ${ }^{57}$ In clinical studies with ESL, the incidence of psychiatric adverse events was low, ${ }^{49}$ and we can expect that $\mathrm{ESL}$, as OXC, may have positive effects in the treatment of 
several psychiatric disturbances even though a meta-analysis failed to show a significant effect of OXC in the treatment of bipolar disorders. ${ }^{58}$

With regards idiosyncratic adverse effects, the incidence of rash, which is the most common idiosyncratic reaction with all AEDs, ${ }^{59}$ seems to be low in patients treated with ESL. This adverse effect occurred in approximately $1 \%$ in all Phase III ESL studies, ${ }^{46-48}$ while it has been reported to be up to $10 \%$ in subjects treated with $\mathrm{OXC}^{60}$ and $11 \%$ in subjects treated with CBZ. ${ }^{61}$

Serious idiosyncratic drug reactions are described with $\mathrm{CBZ}$ and, less often, with $\mathrm{OXC},{ }^{62}$ and recently, pharmacogenetic tests have been suggested to prevent the occurrence of serious cutaneous drug reactions in patients starting selected drug therapies. ${ }^{63-65}$ Since eslicarbazepine is the active metabolite of both ESL and OXC, even though reports on severe adverse drug reactions with ESL are still not available, it may be safe to prescribe these tests in selected populations of predisposed patients before starting this drug. ${ }^{66}$

Hyponatremia (defined as sodium level $<130 \mathrm{mM} / \mathrm{L}$ ) during ESL treatment has been observed in $0.6 \%-1.3 \%$ of patients in premarketing clinical trials. ${ }^{46-49}$ In the Elger et $\mathrm{al}^{46}$ study, mean sodium levels seemed to have an inverse correlation with ESL dosage. However, in the Ben-Menachem et $\mathrm{al}^{48}$ study, three of the four patients with hyponatremia were also taking CBZ. Hyponatremia has also been reported in $1.2 \%$ of patients in an open-label, extension study, ${ }^{51}$ and its values were reported to change from normal to low in $3.2 \%$ of patients in another extension study. ${ }^{52}$ In retrospective studies, hyponatremia has been sporadically reported, but systematic assessments of hematological parameters have not always been performed, and this may have led to underestimation of possible abnormalities. ${ }^{55}$ In the Villanueva et al ${ }^{54}$ study, sodium levels ranging from 116 to $128 \mathrm{mEq} / \mathrm{L}$ were reported in nine patients $(2.7 \%)$, four of whom discontinued treatment. Further studies should assess in more detail the frequency of hyponatremia in patients under treatment with ESL.

Finally, a study specifically aimed at assessing the effect of ESL on the ECG (electrocardiogram) of healthy volunteers failed to show a clinically significant prolongation of the QT interval, ${ }^{67}$ although prolongation of the PR interval has been observed in clinical studies. ${ }^{32}$ Caution should be exercised in patients with medical conditions (eg, low levels of thyroxine, cardiac conduction abnormalities such as second- and third-degree heart block), or when taking concomitant medicinal products known to be associated with PR prolongation. ${ }^{32}$

\section{Experiences in children: efficacy, tolerability, and safety}

There is very limited information on the efficacy and safety of ESL in the pediatric population. To date, there is only one published trial that has analyzed the pharmacokinetics, efficacy, and tolerability of this AED in a small pediatric population of drug-resistant patients. ${ }^{36}$ Doses were from 5 (starting doses) to $30 \mathrm{mg} / \mathrm{kg} / \mathrm{d}$. The pattern of tolerability was dose dependent, similar to what has been observed in adults, and the adverse events were characterized by somnolence, diplopia, vomiting, disequilibrium, and dizziness. In this exploratory study, a dose-dependent decrease in seizure frequency was generally observed.

Few adolescents with focal epilepsies have also been treated with ESL in open studies. ${ }^{55}$

A double-blind, placebo-controlled parallel group trial on children aged 2 to less than 18 years with drug-resistant partial-onset seizures has been completed (NCT 00988156, BIA-2093-305) but not yet published. ${ }^{13}$

A low-dose formulation and/or an oral suspension formulation should be made available for pediatric patients in the near future. ${ }^{13}$

\section{Conclusion and future perspectives}

Some experimental and clinical findings seem to indicate that in respect to OXC, ESL might share similar efficacy but with a more favorable tolerability profile.

Fluctuations of drug levels in plasma affect susceptibility to some AEs, ${ }^{68}$ as it has been clearly demonstrated with CBZ. ${ }^{69}$ Although much less data are available for OXC, in this case it has been observed that several dose-dependent neurological adverse effects occur intermittently and appear almost always a few hours following drug administration. ${ }^{70}$ Since these adverse effects are time-locked with OXC peak concentration, it seems reasonable to suspect that these are caused by $\mathrm{OXC}$ and not by the active metabolite eslicarbazepine, the levels of which increase more slowly. ${ }^{43}$ These kinetic characteristics are lacking after the administration of ESL, which is directly metabolized to eslicarbazepine. ${ }^{11}$

Although data from clinical studies do not allow a precise assessment of efficacy, ${ }^{71}$ tolerability, as assessed by the percentage of patients with some typical dose-dependent, treatment-emergent, neurological adverse events, has been found to be better than that observed with OXC. ${ }^{56}$ Further studies are needed to confirm this finding.

In future, this agent might be also used in patients with newly diagnosed partial-onset seizures and other neurological 
and psychiatric disorders, such as neuropathic pain, migraine, and mania. Indeed, clinical studies aimed at assessing the efficacy of ESL in these fields are ongoing.

\section{Funding}

The authors received no funding for this study. FG is supported by a grant by "Ente Cassa di Risparmio di Firenze."

\section{Disclosure}

GZ has received speaker's or consultancy fees from EISAI, Jansen-Cilag, Sanofi-Aventis, and UCB Pharma. AV has received a grant from Viropharm. MC has received speaker's fees from EBNeuro, Lundbeck, and UCB Pharma. The authors report no other conflicts of interest in this work.

\section{References}

1. Sander JW. The epidemiology of epilepsy revisited. Curr Opin Neurol. 2003;16:165-170.

2. Kwan P, Brodie MJ. Early identification of refractory epilepsy. N Engl J Med. 2000;342:314-319.

3. Loiseau P. Tolerability of newer and older anticonvulsants: a comparative review. CNS Drugs. 1996;6(2):148-166.

4. Perucca P, Gilliam FG. Adverse effects of antiepileptic drugs. Lancet Neurol. 2012;11(9):792-802.

5. Perucca P, Carter J, Vahle V, Gilliam FG. Adverse antiepileptic drug effects: toward a clinically and neurobiologically relevant taxonomy. Neurology. 2009;72(14):1223-1229.

6. Zaccara G, Perucca P. Prevention and management of side-effects of antiepileptic drugs. In: Shorvon SD, Perucca E, editors. The Treatment of Epilepsy. 3rd ed. Malden, MA: Blackwell Science Ltd. In press.

7. Beghi E, Messina P, Pupillo E, et al. Satisfaction with antiepileptic drugs in children and adolescents with newly diagnosed and chronic epilepsy. Epilepsy Res. 2012;100:142-151.

8. Witt JA, Elger CE, Helmstaedter C. Which drug-induced side effects would be tolerated in the prospect of seizure control? Epilepsy Behav. 2013;29:141-143.

9. Faught E. Adherence to antiepilepsy drug therapy. Epilepsy Behav. 2012;25(3):297-302.

10. Benes J, Parada A, Figueiredo AA, et al. Anticonvulsivant and sodium channel-blocking properties of novel 10,11-dihydro-5Hdibenz(b, f)azepine-5-carboxamide derivatives. J Med Chem. 1999;42: 2582-2587.

11. Almeida L, Soares-de Silva P. Eslicarbazepine acetate (BIA 2-093). Neurotherapeutics. 2007;4:88-96.

12. Bialer M, Soares-da-Silva P. Pharmacokinetics and drug interactions of eslicarbazepine acetate. Epilepsia. 2012;53(6):935-946.

13. Verrotti A, Loiacono G, Rossi A, Zaccara G. Eslicarbazepine acetate: an update on efficacy and safety in epilepsy. Epilepsy Res. 2014;108(1): $1-10$.

14. Rogawski MA. Principles of antiepileptic drug action. In: Levy RH, Mattson RH, Meldrum BS, Perucca E, editors. Antiepileptic Drugs. 5th ed. Philadelphia, PA: Lippincot Williams \& Wilkins; 2002:3-22.

15. Yamaoka K, Vogel SM, Seyama I. $\mathrm{Na}^{+}$channel pharmacology and molecular mechanisms of gating. Curr Pharm Des. 2006;12: 429-442.

16. Parada A, Soares-da-Silva P. The novel anticonvulsant BIA 2-093 inhibits transmitter release during opening of voltage gated sodium channels: a comparison with carbamazepine and oxcarbazepine. Neurochem Int. 2002;40:435-440.
17. Bonifacio MJ, Sheridan RD, Parada A, Cunha RA, Patmore L, Soaresda-Silva P. Interaction of the novel anticonvulsant, BIA 2-093, with voltage-gated sodium channels: comparison with carbamazepine. Epilepsia. 2001;42:600-608.

18. Brown ME, El-Mallakh RS. Role of eslicarbazepine in the treatment of epilepsy in adult patients with partial-onset seizures. Ther Clin Risk Manag. 2010;6:103-109.

19. Vilin YY, Ruben PC. Slow inactivation in voltage-gated sodium channels: molecular substrates and contributions to channelopathies. Cell Biochem Biophys. 2001;35:171-190.

20. Goldin AL. Mechanisms of sodium channel inactivation. Curr Opin Neurobiol. 2003;13:284-290.

21. Eijkelkamp N, Linley JE, Baker MD, et al. Neurological perspectives on voltage-gated sodium channels. Brain. 2012;135:2585-2612.

22. McCollum IJ, Vilin YY, Spackman E, Fujimoto E, Ruben PC. Negatively charged residues adjacent to IFM motif in the DIII-DIV linker of $\mathrm{hNa}(\mathrm{V}) 1.4$ differentially affect slow inactivation. FEBS Lett. 2003;552:163-169.

23. Chen Y, Yu FH, Surmeier DJ, Scheuer T, Catterall WA. Neuromodulation of $\mathrm{Na}+$ channel slow inactivation via cAMP-dependent protein kinase and protein kinase C. Neuron. 2006;49:409-420.

24. O'Reilly JP, Wang SY, Wang GK. Residue-specific effects on slow inactivation at $\mathrm{V} 787$ in D2-S6 of $\mathrm{Na}(\mathrm{v}) 1.4$ sodium channels. Biophys $J$. 2001;81:2100-2111.

25. Errington AC, Stohr T, Heers C, Lees G. The investigational anticonvulsant lacosamide selectively enhances slow inactivation of voltage-gated sodium channels. Mol Pharmacol. 2008;73:157-169.

26. Hebeisen S, Pires N, Loureiro AI, et al. Eslicarbazepine and the enhancement of slow inactivation of voltage-gated sodium channels: a comparison with carbamazepine, oxcarbazepine and lacosamide. Neuropharmacology. 2015;89:122-135.

27. Sierra-Paredes G, Oreiro-Garcia MT, Vazquez-Illanes MD, SierraMarcuno G. Effect of eslicarbazepine acetate (BIA 2-093) on latrunculin A-induced seizures and extracellular amino acid concentrations in the rat hippocampus. Epilepsy Res. 2007;77(1):36-43.

28. Sierra-Paredes G, Nunez-RodriguezA, Vazquez-LopezA, Oreiro-GarciaT, Sierra-Marcuno G. Anticonvulsant effect of eslicarbazepine acetate (BIA 2-093) on seizures induced by microperfusion of picrotoxin in the hippocampus of freely moving rats. Epilepsy Res. 2006; 72(2-3):140-146.

29. Araujo IM, Ambrosio AF, Leal EC, et al. Neurotoxicity induced by antiepileptic drugs in cultured hippocampal neurons: a comparative study between carbamazepine, oxcarbazepine, and two new putative antiepileptic drugs, BIA 2-024 and BIA 2-093. Epilepsia. 2004;45(12): $1498-1505$.

30. Vaz-de-Silva M, Nunes T, Soares E, et al. Eslicarbazepine acetate pharmacokinetics after single and repeated doses in healthy subjects. Epilepsia. 2005;46:191.

31. Perucca E, Elger C, Halasz P, Falcao A, Almeida L, Soares-da-Silva P. Pharmacokinetics of eslicarbazepine acetate at steady-state in adults with partial-onset seizures. Epilepsy Res. 2011;96:132-139.

32. European Medicines Agency. Zebinix1 (eslicarbazepine acetate tablets): summary of product characteristics [online]. Available from: http:// www.ema.europa.eu/docs/en_GB/document_library/EPAR___Product_ Information/human/000988/WC500047225.pdf. Accessed November 6, 2014.

33. Hainzl D, Parada A, Soares-da-Silva P. Metabolism of two new antiepileptic drugs and their principal metabolites $\mathrm{S}(+)$ - and $\mathrm{R}(-)-10,11-$ dihydro-10-hydroxy carbamazepine. Epilepsy Res. 2001;44:197-206.

34. Silveira P, Falcao A, Almeida L, Maia J, Soares-da-Silva P. BIA 2-093 pharmacokinetics in healthy elderly subjects. Epilepsia. 2004;45 (Suppl 8):157.

35. Elger C, Bialer M, Falcão A, et al. Pharmacokinetics and tolerability of eslicarbazepine acetate and oxcarbazepine at steady state in healthy volunteers. Epilepsia. 2013;54(8):1453-1461. 
36. Almeida L, Minciu I, Nunes T, et al. Pharmacokinetics, efficacy, and tolerability of eslicarbazepine acetate in children and adolescents with epilepsy. J Clin Pharmacol. 2008;48:966-977.

37. Falcão A, Maia J, Almeida L, Mazur D, Gellert M, Soares-da-Silva P. Effect of gender on the pharmacokinetics of eslicarbazepine acetate (BIA 2-093), a new voltage-gated sodium channel blocker. Biopharm Drug Dispos. 2007;28:249-256.

38. Maia J, Almeida L, Falcao A, et al. Effect of renal impairment on the pharmacokinetics of eslicarbazepine acetate. Int J Clin Pharmacol Ther. 2008;46(3):119-130.

39. Almeida L, Potgieter JH, Maia J, Potgieter MA, Mota F, Soares-da-Silva P. Pharmacokinetics of eslicarbazepine acetate in patients with moderate hepatic impairment. Eur J Clin Pharmacol. 2008;64:267-273.

40. Falcão A, Fuseau E, Nunes T, Almeida L, Soares-da-Silva P. Pharmacokinetics, drug interactions and exposure-response relationship of eslicarbazepine acetate in adult patients with partial-onset seizures: population pharmacokinetic and pharmacokinetic/pharmacodynamic analyses. CNS Drugs. 2012;26(1):79-91.

41. Falcão A, Vaz-da-Silva M, Gama H, Nunes T, Almeida L, Soares-daSilva P. Effect of eslicarbazepine acetate on the pharmacokinetics of a combined ethinylestradiol/levonorgestrel oral contraceptive in healthy women. Epilepsy Res. 2013;105(3):368-376.

42. Vaz-da-Silva M, Almeida L, Falcão A, et al. Effect of eslicarbazepine acetate on the steady-state pharmacokinetics and pharmacodynamics of warfarin in healthy subjects during a three-stage, open-label, multipledose, single-period study. Clin Ther. 2010;32(1):179-192.

43. FaughtE. Oxcarbazepine. In: Shorvon SD, PeruccaE, FishDR, Dodson WE, editors. The Treatment of Epilepsy. 2nd ed. Malden, MA: Blackwell Science Ltd; 2004:451-460.

44. Nunes T, Rocha JF, Falcão A, Almeida L, Soares-da-Silva P. Steadystate plasma and cerebrospinal fluid pharmacokinetics and tolerability of eslicarbazepine acetate and oxcarbazepine in healthy volunteers. Epilepsia. 2013;54(1):108-116.

45. Elger C, Bialer M, Cramer JA, Maia J, Almeida L, Soares-da-Silva P. Eslicarbazepine acetate: a double-blind, add-on, placebo-controlled exploratory trial in adult patients with partial-onset seizures. Epilepsia. 2007;48(3):497-504.

46. Elger C, Halász P, Maia J, Almeida L, Soares-da-Silva P; BIA-2093301 Investigators Study Group. Efficacy and safety of eslicarbazepine acetate as adjunctive treatment in adults with refractory partial-onset seizures: a randomized, double-blind, placebo-controlled, parallel-group phase III study. Epilepsia. 2009;50(3):454-463.

47. Gil-Nagel A, Lopes-Lima J, Almeida L, Maia J, Soares-da-Silva P; BIA-2093-303 Investigators Study Group. Efficacy and safety of 800 and $1,200 \mathrm{mg}$ eslicarbazepine acetate as adjunctive treatment in adults with refractory partial-onset seizures. Acta Neurol Scand. 2009;120(5):281-287.

48. Ben-Menachem E, Gabbai AA, Hufnagel A, Maia J, Almeida L, Soares-da-Silva P. Eslicarbazepine acetate as adjunctive therapy in adult patients with partial epilepsy. Epilepsy Res. 2010;89(2-3):278-285.

49. Gil-Nagel A, Elger C, Ben-Menachem E, et al. Efficacy and safety of eslicarbazepine acetate as add-on treatment in patients with focal-onset seizures: integrated analysis of pooled data from double-blind phase III clinical studies. Epilepsia. 2013;54(1):98-107.

50. Halász P, Cramer JA, Hodoba D, et al; BIA-2093-301 Study Group. Long-term efficacy and safety of eslicarbazepine acetate: results of a 1-year open-label extension study in partial-onset seizures in adults with epilepsy. Epilepsia. 2010;51(10):1963-1969.

51. Hufnagel A, Ben-Menachem E, Gabbai AA, Falcão A, Almeida L, Soares-da-Silva P. Long-term safety and efficacy of eslicarbazepine acetate as adjunctive therapy in the treatment of partial-onset seizures in adults with epilepsy: results of a 1-year open-label extension study. Epilepsy Res. 2013;03(2-3):262-269.

52. Massot A, Vivanco R, Principe A, Roquer J, Rocamora R. Postauthorisation study of eslicarbazepine as treatment for drug-resistant epilepsy: preliminary results. Neurologia. 2014;29(2):94-101.
53. Serrano-Castro PJ, Payan-Ortiz M, Cimadevilla JM, Quiroga-Subirana P, Fernandez-Perez J. Eslicarbazepine acetate in clinical practice. Efficacy and safety results. Rev Neurol. 2013;56:309-314.

54. Villanueva V, Serratosa JM, Guillamón E, et al. Long-term safety and efficacy of eslicarbazepine acetate in patients with focal seizures: results of the 1-year ESLIBASE retrospective study. Epilepsy Res. 2014;108(7):1243-1252.

55. Correia FD, Freitas J, Magalhães R, et al. Two-year follow-up with eslicarbazepine acetate: a consecutive, retrospective, observational study. Epilepsy Res. 2014;108(8):1399-1405.

56. Zaccara G, Giovannelli F, Maratea D, Fadda V, Verrotti A. Neurological adverse events of new generation sodium blocker antiepileptic drugs. Meta-analysis of randomized, double-blinded studies with eslicarbazepine acetate, lacosamide and oxcarbazepine. Seizure. 2013;22(7): 528-536.

57. Eddy CM, Rickards HE, Cavanna AE. Behavioral adverse effects of antiepileptic drugs in epilepsy. Clin Psychopharmacol. 2012;32(3): 362-375.

58. Vasudev A, Macritchie K, Vasudev K, Watson S, Geddes J, Young AH. Oxcarbazepine for acute affective episodes in bipolar disorder. Cochrane Database Syst Rev. 2011;(12):CD004857.

59. Zaccara G, Franciotta D, Perucca E. Idiosyncratic adverse reactions to antiepileptic drugs. Epilepsia. 2007;48(7):1223-1244.

60. Shorvon SD. Oxcarbazepine: a review. Seizure. 2000;9:75-79

61. Mattson RH, Cramer JA, Collins JF, et al. A comparison of valproate with carbamazepine for the treatment of partial seizures and secondarily generalised tonic-clonic seizures in adults. $N$ Engl J Med. 1992; 327:765-771.

62. Tennis P, Stern RS. Risk of serious cutaneous disorders after initiation of use of phenytoin, carbamazepine, or sodium valproate: a record linkage study. Neurology. 1997;49(2):542-546.

63. Wang L, McLeod HL, Weinshilboum RM. Genomics and drug response. N Engl J Med. 2011;364(12):1144-1153.

64. Chen P, Lin JJ, Lu CS, et al. Carbamazepine-induced toxic effects and HLA-B*1502 screening in Taiwan. $N$ Engl J Med. 2011;364(12): 1126-1133.

65. McCormack M, Alfirevic A, Bourgeois S, et al. HLA-A*3101 and carbamazepine-induced hypersensitivity reactions in Europeans. N Engl J Med. 2011;364(12):1134-1143.

66. Kaniwa N, Saito Y. The risk of cutaneous adverse reactions among patients with the HLA-A* 31:01 allele who are given carbamazepine, oxcarbazepine or eslicarbazepine: a perspective review. Ther Adv Drug Saf. 2013;4(6):246-253.

67. Vaz-Da-Silva M, Nunes T, Almeida L, Gutierrez MJ, Litwin JS, Soares-Da-Silva P. Evaluation of eslicarbazepine acetate on cardiac repolarization in a thorough QT/QTc study. J Clin Pharmacol. 2012; 52(2):222-233.

68. Zaccara G, Cincotta M, Borgheresi A, Balestrieri F. Adverse motor effects induced by antiepileptic drugs. Epileptic Disord. 2004;6(3): $153-168$.

69. Tothfalusi L, Speidl S, Endrenyil L. Exposure-response analysis reveals that clinically important toxicity difference can exist between bioequivalent carbamazepine tablets. Br J Clin Pharmacol. 2008;65(1): $110-122$.

70. Striano S, Striano P, Di Nocera P, et al. Relationship between serum mono-hydroxy-carbazepine concentrations and adverse effects in patients with epilepsy on high-dose oxcarbazepine therapy. Epilepsy Res. 2006;69(2):170-176.

71. Zaccara G, Giovannelli F, Bell GS, Sander JW. Network meta-analyses of antiepileptic drug efficacy and tolerability in drug-resistant focal epilepsies: a clinical perspective. Eur J Clin Pharmacol. 2014;70(6): 647-654. 


\section{Publish your work in this journal}

Drug Design, Development and Therapy is an international, peerreviewed open-access journal that spans the spectrum of drug design and development through to clinical applications. Clinical outcomes, patient safety, and programs for the development and effective, safe, and sustained use of medicines are a feature of the journal, which has also been accepted for indexing on PubMed Central. The manuscript management system is completely online and includes a very quick and fair peer-review system, which is all easy to use. Visit http://www.dovepress.com/testimonials.php to read real quotes from published authors.

Submit your manuscript here: http://www.dovepress.com/drug-design-development-and-therapy-journal 